\title{
ON HALF-SPACE SOLUTIONS OF A MODIFIED HEAT EQUATION*
}

\author{
BY \\ D. E. AMOS \\ Sandia Laboratory, Albuquerque, New Mexico
}

In a recent paper on the foundations of thermodynamics, Gurtin and Williams [8] showed that under certain physically reasonable hypotheses the first and second laws of thermodynamics (for rigid heat conductors) are

$$
\begin{aligned}
& \frac{d}{d t} \int_{R} e d v=-\int_{\partial R} \mathbf{q} \cdot \mathbf{n} d a+\int_{R} r d v \\
& \frac{d}{d t} \int_{R} \eta d v \geq-\int_{\partial R} \frac{\mathbf{q} \cdot \mathbf{n}}{\varphi} d a+\int_{R} \frac{r}{\theta} d v
\end{aligned}
$$

where $e$ is the internal energy (per unit volume), q the heat flux vector, $r$ the heat supply (per unit volume), $\eta$ the entropy (per unit volume), $\varphi$ the conductive temperature, and $\theta$ the thermodynamic temperature. They [8] also proved that in general the two temperatures $\varphi$ and $\theta$ are equal. Subsequently, Chen and Gurtin [2] formulated a theory of materials in which the two temperatures were unequal. They showed that for an isotropic material the linearized version of the energy equation takes the form

$$
c \dot{\varphi}=k \Delta \varphi+c \epsilon \Delta \dot{\varphi}+r
$$

where $c, k$, and $\epsilon$ are constants, $c$ being the specific heat, $k$ the conductivity and $\epsilon$ the temperature discrepancy factor since the thermodynamic temperature is related to the conductive temperature by the formula $\theta=\varphi-\epsilon \Delta \varphi$. Furthermore, they showed that if $k$ is positive, then $\epsilon \geq 0$.

Eq. (1) also arises in the theory developed by Barenblatt, Zheltov, and Kochina [1] for flow through fissured rock. Its one-dimensional counterpart was derived by Coleman and Noll [4] as governing the simple shearing motion of a fluid of second grade.

The purpose of the analysis below is to contrast the one-dimensional case $r=0$, $\epsilon>0$ with the classical case, $r=\epsilon=0$, for the half plane $x \geq 0$ with boundary conditions related to

$$
\begin{aligned}
& \varphi(x, 0)=0 \quad x>0 \\
& \varphi(0, t)=1 \quad t>0 .
\end{aligned}
$$

The boundary value problem. The boundary value problem (Eq. (1) rescaled)

$$
\begin{aligned}
& u_{t}=u_{x x}+\epsilon u_{x x t} \quad x>0, \quad t>0, \quad \epsilon \geq 0, \\
& u(x, 0)=0 \quad x>0, \\
& u(0, t)=1 \quad t>0, \\
& u \text { bounded, }
\end{aligned}
$$

${ }^{*}$ Received May 5, 1968; revised version received October 17, 1968. This work was supported by the United States Atomic Energy Commission. 
has a formal solution by separation of variables given by

$$
u_{1}(x, t)=1-\frac{2}{\pi} \int_{0}^{\infty} e^{-v^{2} t /\left(1+\epsilon v^{2}\right)} \frac{\sin v x}{v} d v .
$$

This function formally satisfies the conditions of the problem, but a change of variables $w=x v, x>0$ gives

$$
u_{1}(x, t)=1-\frac{2}{\pi} \int_{0}^{\infty} e^{-w^{2} t /\left(x^{2}+\epsilon w^{2}\right)} \frac{\sin w}{w} d w
$$

which shows that the solution is discontinuous at $x=0$ :

$$
\lim _{x \rightarrow 0+} u_{1}(x, t)=1-e^{-t / \epsilon} \neq 1 \quad t>0
$$

(justification of the limiting process is given below). This result is in agreement with [1] where, according to singular surface theory, the jump in the discontinuity decays according to the right side of this relation.

If one attempts the construction of a generalized solution by introducing the initial condition

$$
u(x, 0)=e^{-\alpha x} \quad \alpha>0, x>0
$$

in place of

$$
u(x, 0)=0,
$$

the resultant solution as $\alpha \rightarrow \infty$ also goes to (3) or (4). On the other hand, specification of analytic data in the form

$$
u(0, t)=1-e^{-\alpha t} \quad \alpha>0, \quad t \geq 0
$$

gives for $\alpha \rightarrow \infty$

$$
u_{2}(x, t)=1-\frac{2}{\pi} \int_{0}^{\infty} e^{-v^{2} t /\left(1+\epsilon v^{2}\right)} \frac{\sin v x}{v\left(1+\epsilon v^{2}\right)} d v
$$

or

$$
u_{2}(x, t)=1-\frac{2 x^{2}}{\pi} \int_{0}^{\infty} e^{-w^{2} t /\left(x^{2}+\epsilon w^{2}\right)} \frac{\sin w}{w\left(x^{2}+\epsilon w^{2}\right)} d w
$$

which does not satisfy $u(x, 0)=0$ for $x \geq 0$, but

$$
\lim _{t \rightarrow 0+} u_{2}(x, t)=e^{-x / \epsilon^{1 / 2}} \text {. }
$$

The result in applying the Laplace transform to (2) under the assumptions that $u(x, t)$ and $u_{x x}(x, t)$ are continuous at $t=0$ is (5); on the other hand, the formal Fourier sine transform solution with the assumptions that the $u$ and $u_{x} \rightarrow 0$ as $x \rightarrow \infty$ gives (3). These two results have important implications for a numerical solution. In a physical application one may choose the results of one of these solutions because of experimental correlation with theory for longer times and distances even though a detailed knowledge of the mechanism at $x=0$ and $t=0$ may be lacking.

Each of the functions listed in (3) and (5) is continuous in $\epsilon$ for fixed $x$ and $t$. In particular, for $\epsilon=0$ we have 


$$
\begin{aligned}
u_{0}(x, t) & =1-\frac{2}{\pi} \int_{0}^{\infty} e^{-v^{2} t} \frac{\sin v x}{v} d v \\
& =\operatorname{erfc}\left(\frac{x}{2 t^{1 / 2}}\right)
\end{aligned}
$$

which is the solution of the classical heat conduction problem. The uniform convergence on $[0, \bar{\epsilon}]$ can be seen by applying the Dirichlet test $[11, \text { p. } 23]^{1}$ for conditionally convergent integrals in the form

$$
\int_{0}^{\infty} \phi(v, \epsilon) f(v, \epsilon) d v
$$

where

$$
\begin{aligned}
\phi(v, \epsilon) & =\frac{e^{-v^{2} t /\left(x^{2}+\epsilon^{2}\right)}}{1+v} \text { or } \frac{x^{2} e^{-v^{2} t /\left(x^{2}+\epsilon v^{2}\right)}}{\left(x^{2}+\epsilon v^{2}\right)(1+v)}, \\
f(v, \epsilon) & =\frac{(1+v)}{v} \sin v .
\end{aligned}
$$

Here $\partial \phi / \partial v<0$ is continuous in $v$ for fixed $\epsilon, F(v)=\int_{0}^{v} f(w, \epsilon) d w$ is bounded independent of $v$ and $\epsilon$, and $\phi(v, \epsilon) \rightarrow 0$ monotonically in $v$ and uniformly on $[0, \bar{\epsilon}]$ as $v \rightarrow \infty$ since $\partial \phi / \partial v<0$ and $0<\phi<1 /(1+v)$.

Notice that this same analysis applies for $t$ or $x$ on $[0, \bar{t}],[0, \bar{x}]$ or $[0, \bar{x}] \times[0, t]$.

Series representations of (4) and (6). In this section we develop two series for (4) and (6) which tend to complement each other in numerical evaluation. These series consist of positive terms involving functions for which good numerical techniques are available for computation. We start with (4) and write the exponential in the integral in the form

$$
I=\int_{0}^{\infty} e^{-v^{2} T /\left(X^{2}+v^{2}\right)} \frac{\sin v}{v} d v=\frac{\pi}{2}+\sum_{n=1}^{\infty} \frac{(-1)^{n} T^{n}}{n !} \int_{0}^{\infty} \frac{v^{2 n-1} \sin v}{\left(X^{2}+v^{2}\right)^{n}} d v
$$

where $X=x / \epsilon^{1 / 2}, T=t / \epsilon$. The Fourier transform is available from tables [5, p. 68],

$$
\int_{0}^{\infty} \frac{v^{2 m+1} \sin v}{\left(z+v^{2}\right)^{n}} d v=\frac{(-1)^{m+n-1} \pi / 2}{(n-1) !} \frac{d^{n-1}}{d z^{n-1}}\left(z^{m} e^{-z^{1 / 2}}\right) \quad 0 \leq 2 m \leq 2 n-2,
$$

and we apply this with $m=n-1$. The Leibnitz rule for differentiation of a product

$$
\frac{d^{(n)}}{d z^{(n)}}(u v)=\sum_{k=0}^{n}\left(\begin{array}{l}
n \\
k
\end{array}\right) u^{(k)} v^{(n-k)}
$$

will expand the right side into a sum in terms of

$$
\begin{aligned}
\left(z^{n-1}\right)^{(n-k-1)} & =(n-1)(n-2) \cdots(k+1) z^{k} \quad k=0,1, \cdots n-2, \\
& =z^{n-1} \quad k=n-1,
\end{aligned}
$$

and the derivatives of $e^{-z^{1 / 2}}$ :

${ }^{2}$ Continuity of $\partial \varphi / \partial v$ can be relaxed to continuity in $v$ for fixed $\epsilon$ in order to apply the proof. 


$$
\begin{aligned}
& \left(e^{-z^{1 / 2}}\right)^{(1)}=-e^{-z^{1 / 2} /\left(2 z^{1 / 2}\right)} \\
& \left(e^{-z^{1 / 2}}\right)^{(2)}=e^{-z^{1 / 2}}\left(\frac{1}{2 z^{1 / 2}}\right)^{2}\left(1+\frac{1}{z^{1 / 2}}\right)
\end{aligned}
$$

or, more generally,

$$
\left(e^{-z^{1 / 2}}\right)^{(k)}=Q_{2 k-1}\left(\frac{1}{z^{1 / 2}}\right) e^{-z^{1 / 2}}
$$

Now,

$$
\left(e^{-z^{1 / 2}}\right)^{(k+1)}=e^{-z^{1 / 2}} Q_{2 k+1}\left(\frac{1}{z^{1 / 2}}\right)=\frac{e^{-z^{1 / 2}}}{2 z^{1 / 2}}\left[-Q_{2 k-1}\left(\frac{1}{z^{1 / 2}}\right)-\frac{1}{z} Q_{2 k-1}^{\prime}\left(\frac{1}{z^{1 / 2}}\right)\right]
$$

and the polynomials $Q_{2 k-1}$ have the recurrence relation

$$
Q_{2 k+1}(x)=-\frac{x}{2} Q_{2 k-1}(x)-\frac{x^{3}}{2} Q_{2 k-1}^{\prime}(x) \quad k=1,2, \cdots
$$

where $Q_{1}(x)=-x / 2$. A slight change in notation,

$$
Q_{2 k-1}(x)=\frac{(-x)^{k}}{2^{k}} P_{k-1}(x) \quad k=1,2, \cdots,
$$

gives

$$
\left(e^{-z^{1 / 2}}\right)^{(k)}=\frac{(-x)^{k}}{2^{k}} P_{k-1}(x) e^{-z^{1 / 2}}
$$

with $x=1 / z^{1 / 2}, P_{-1}(x) \equiv 1, P_{0}(x) \equiv 1$ and

$$
P_{k}(x)=(1+k x) P_{k-1}(x)+x^{2} P_{k-1}^{\prime}(x) \quad k=1,2, \cdots .
$$

The right side of (7) takes the form

$$
\frac{\pi}{2}(n-1) ! e^{-z^{1 / 2}} \sum_{k=0}^{n-1} \frac{(-1)^{k}}{(n-k-1) !(k !)^{2}}\left(\frac{z^{1 / 2}}{2}\right)^{k} P_{k-1}\left(\frac{1}{z^{1 / 2}}\right)
$$

and with $P_{-1}(x) \equiv 1$,

$$
\begin{aligned}
u_{1}(x, t) & =-e^{-X} \sum_{n=1}^{\infty} \frac{(-1)^{n} T^{n}}{n} \sum_{k=0}^{n-1} \frac{(-1)^{k}}{(n-k-1) !} \frac{\left(\frac{X}{2}\right)^{k}}{(k !)^{2}} P_{k-1}\left(\frac{1}{X}\right) \\
& =-e^{-X} \sum_{k=0}^{\infty} \frac{(-1)^{k}}{(k !)^{2}}\left(\frac{X}{2}\right)^{k} P_{k-1}\left(\frac{1}{X}\right) \sum_{n=k+1}^{\infty} \frac{(-1)^{n} T^{n}}{n(n-k-1) !}
\end{aligned}
$$

after changing orders of summation. A shift of index in the second sum, $n=k+1+p$, gives a series which is identifiable as an incomplete gamma function,

$$
(-1)^{k+1} \sum_{p=0}^{\infty} \frac{(-1)^{p} T^{p+k+1}}{p !(p+k+1)}=(-1)^{k+1} \int_{0}^{T} e^{-v} v^{k} d v
$$

The final form for $u_{1}$ is 


$$
u_{1}(x, t)=e^{-x} \sum_{k=0}^{\infty} \frac{X^{k}}{2^{k} k !} P_{k-1}\left(\frac{1}{X}\right) \gamma(k+1, T)
$$

where $P_{-1}(x) \equiv 1$ and

$$
\gamma(k+1, T)=\frac{1}{\Gamma(k+1)} \int_{0}^{T} e^{-v} v^{k} d v .
$$

Numerical values (to a specified number of significant figures) for these gamma functions can be generated very efficiently by the procedure described in [6].

An explicit expression for $P_{k}(x)$ is easily obtained by examining the transform [5, p. 67]

$$
\int_{0}^{\infty} \frac{v \sin v}{\left(z+v^{2}\right)^{n}} d v=\frac{e^{-z^{1 / 2}} \pi / 2}{(n+1) !\left(2 z^{1 / 2}\right)^{2 n-3}} \sum_{m=0}^{n-2} \frac{(2 n-m-4) !}{m !(n-m-2) !}\left(2 z^{1 / 2}\right)^{m}
$$

and comparing this with (7) for $m=0$,

$$
\int_{0}^{\infty} \frac{v \sin v}{\left(z+v^{2}\right)^{n}} d v=\frac{(-1)^{n-1} \pi / 2}{(n-1) !}\left(\frac{-1}{2 z^{1 / 2}}\right)^{n-1} P_{n-2}\left(\frac{1}{z^{1 / 2}}\right) e^{-z^{1 / 2}} .
$$

Then, by summing in the reverse direction and replacing $n-2$ by $n$,

$$
P_{n}(x)=\sum_{m=0}^{n} \frac{(n+m) !}{m !(n-m) !}\left(\frac{x}{2}\right)^{m} .
$$

The terms

$$
F_{k-1}(X)=\frac{X^{k}}{2^{k} k !} P_{k-1}\left(\frac{1}{X}\right) \quad k \geq 1
$$

remain well scaled, even for very large $X$, but some rearrangement is necessary to ensure that all factors remain scaled:

$$
F_{k-1}(X)=\frac{1}{2^{k} k !} \sum_{r=1}^{k} \frac{(2 k-r-1) !}{(k-r) !(r-1) !} \frac{X^{r}}{2^{k-r}}=\sum_{r=1}^{k} A_{r}
$$

where

$$
A_{1}=\frac{k X(2 k-2) !}{2^{2 k-1}(k !)^{2}}=\frac{X}{2 \pi^{1 / 2}} \frac{\Gamma(k-1 / 2)}{\Gamma(k+1)} \sim \frac{X}{2 \pi^{1 / 2} k^{3 / 2}}
$$

and

$$
A_{r+1}=A_{r} \frac{(k-r)(2 X)}{r(2 k-r-1)}, \quad r=1,2, \cdots, k-1 .
$$

The fact that $u_{1}(x, t) \rightarrow 1$ as $T \rightarrow \infty$ is shown by exchanging the order of summation in (8) with the aid of (9) and noting that

$$
\sum_{p=0}^{\infty} \frac{(2 p+r-1) !}{2^{2 p+r} p !(r+p) !}=\int_{0}^{\infty} \frac{e^{-v} I_{r}(v)}{v} d v=\frac{1}{r} .
$$

A second series for (4) can be obtained in which $X$ and $T$ essentially exchange roles. This is done by adding and subtracting $e^{-T}$ on the right of (4) in the form

$$
e^{-T}=\frac{2}{\pi} \int_{0}^{\infty} \frac{\sin v}{v} e^{-T} d v
$$


Then

$$
u_{1}(x, t)=1-e^{-T}-\frac{2 e^{-T}}{\pi} \int_{0}^{\infty}\left[e^{X^{2} T /\left(X^{2}+v^{2}\right)}-1\right] \frac{\sin v}{v} d v .
$$

Expansion of the exponential this time leads to

$$
u_{1}(x, t)=1-e^{-T}-\frac{2 e^{-T}}{\pi} \sum_{n=1}^{\infty} \frac{\left(X^{2} T\right)^{n}}{n !} \int_{0}^{\infty} \frac{\sin v}{v\left(X^{2}+v^{2}\right)^{n}} d v .
$$

This latter integral is evaluated by integrating the transform [5, p. 14]

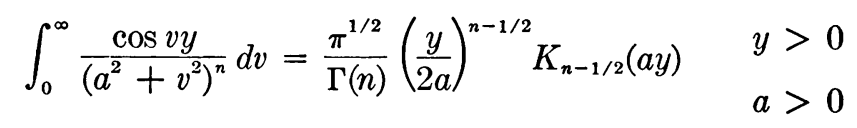

on $0<y \leq 1$ after noting that

$$
K_{n-1 / 2}(z)=\left(\frac{\pi}{2 z}\right)^{1 / 2} e^{-z} \sum_{m=0}^{n-1} \frac{\Gamma(m+n)}{m ! \Gamma(n-m)} \frac{1}{(2 z)^{m}} .
$$

The result is

$$
\int_{0}^{\infty} \frac{\sin v d v}{v\left(X^{2}+v^{2}\right)^{n}}=\frac{\pi}{X^{2 n}} \sum_{m=0}^{n-1} \frac{\Gamma(m+n)}{m ! \Gamma(n) 2^{m+n}} \gamma(n-m, X)
$$

and

$$
u_{1}(x, t)=1-e^{-T}-2 e^{-T} \sum_{n=1}^{\infty} \frac{T^{n}}{n !} \sum_{m=0}^{n-1} \frac{\Gamma(m+n) \gamma(n-m, X)}{2^{m+n} m ! \Gamma(n)} .
$$

$u_{1 x x}(x, t)$ is also needed to compute the thermodynamic temperature for the results in [2]. This can be done by using

$$
\begin{aligned}
u_{1 x x}(x, t)=\frac{e^{-X}}{\epsilon}\left[\gamma(1, T)+\frac{(X-2)}{2}\right. & \gamma(2, T) \\
& \left.\quad+\sum_{k=2}^{\infty} \gamma(k+1, T)\left[F_{k-1}(X)-2 G_{k-1}(X)+H_{k-1}(X)\right]\right]
\end{aligned}
$$

where

$$
\begin{aligned}
& F_{k-1}(X)=\sum_{r=1}^{k} A_{r}, \\
& G_{k-1}(X)=\frac{1}{X} \sum_{r=1}^{k} r A_{r}, \\
& H_{k-1}(X)=\frac{1}{X^{2}} \sum_{r=1}^{k} r(r-1) A_{r},
\end{aligned}
$$

or

$$
u_{1 x x}(x, t)=\frac{-2 e^{-T}}{\epsilon} \sum_{n=1}^{\infty} \frac{T^{n}}{n !} \sum_{m=0}^{n-1} \frac{\Gamma(m+n) g(n-m, X)}{2^{m+n} m ! \Gamma(n)}
$$

where $g(n-m, X)=X^{n-m-2} e^{-x}[n-m-1-X] / \Gamma(n-m)$. Numerical results based upon (8) and (11) and the corresponding formulae for $u_{1 x x}(x, t)$ are shown in [2]. 
If we note

$$
1-e^{-x}=\frac{2 X^{2}}{\pi} \int_{0}^{\infty} \frac{\sin v}{v\left(X^{2}+v^{2}\right)} d v
$$

from (10), expressions similar to (8) and (11) can be written down for $u_{2}(x, t)$. They are

$$
u_{2}(x, t)=e^{-X}+e^{-x} \sum_{k=1}^{\infty} \frac{X^{k}}{2^{k} k !} P_{k-1}\left(\frac{1}{X}\right) \gamma(k, T)
$$

and

$$
u_{2}(x, t)=1-e^{-T}\left(1-e^{-X}\right)-2 e^{-T} \sum_{n=1}^{\infty} \frac{T^{n}}{n !} \sum_{m=0}^{n} \frac{\Gamma(m+n+1) \gamma(n-m+1, X)}{m ! \Gamma(n+1) 2^{m+n+1}}
$$

Asymptotics for $\epsilon \rightarrow 0$. Since (1) was obtained by linearizing the heat conduction theory in [2], one expects $\epsilon$ to be small in a physical application. We therefore develop the asymptotic expressions in $\epsilon$ for $u_{1}(x, t)$ and $u_{2}(x, t)$. This we do by taking the Laplace transform of expressions (4) and (6), expanding into power series in $\epsilon$ and inverting them term by term. Then for $u_{1}(x, t)$ we have

$$
\begin{aligned}
\bar{u}_{1}(x, s)= & \frac{1}{s}-\frac{2}{\pi} \int_{0}^{\infty} \frac{x^{2}+\epsilon v^{2}}{x^{2} s+s \epsilon v^{2}+v^{2}} \frac{\sin v}{v} d v=\frac{e^{-q x}}{s(1+\epsilon s)} \\
= & \frac{1}{s}-\frac{2}{\pi} \int_{0}^{\infty} \frac{\sin v}{v\left(s+v^{2} / x^{2}\right)} d v \\
& \quad+\frac{2}{\pi} \sum_{n=1}^{\infty} \frac{(-1)^{n} \epsilon^{n}}{x^{2 n+2}} \int_{0}^{\infty} \frac{v^{2 n+1} s^{n-1} \sin v}{\left(s+v^{2} / x^{2}\right)^{n+1}} d v
\end{aligned}
$$

where

$$
q=\left(\frac{s}{1+\epsilon s}\right)^{1 / 2}
$$

Term by term inversion gives [5, p. 175]

$$
u_{1}(x, t)=\operatorname{erfc}\left(\frac{x}{2 t^{1 / 2}}\right)+\sum_{n=1}^{\infty} \frac{(-1)^{n} \epsilon^{n} t}{n x^{2 n+2}} \int_{0}^{\infty} v^{2 n+1} e^{-v^{2} t / x^{2}} L_{n-1}^{(1)}\left(\frac{v^{2} t}{x^{2}}\right) \sin v d v
$$

The Laguerre polynomial,

$$
\frac{1}{n} L_{n-1}^{(1)}\left(\frac{v^{2} t}{x^{2}}\right)=\sum_{k=0}^{n-1} \frac{(-n+1)_{k}}{(k+1) !} \frac{\left(t / x^{2}\right)^{k}}{k !} v^{2 k}
$$

together with

$$
\int_{0}^{\infty} v^{2 n+2 k+1} e^{-v^{2} t / x^{2}} \sin v d v=\frac{(-1)^{n+k} \pi^{1 / 2}}{2^{n+k+3 / 2}}\left(\frac{x^{2}}{t}\right)^{n+k+1} e^{-x^{2} /(4 t)} H e_{2 n+2 k+1}\left(\frac{x}{(2 t)^{1 / 2}}\right),
$$

gives

$$
u_{1}(x, t)=\operatorname{erfc}\left(\frac{x}{2 t^{1 / 2}}\right)+\frac{e^{-x^{2 /(4 t)}}}{(2 \pi)^{1 / 2}} \sum_{n=1}^{\infty}\left(\frac{\epsilon}{2 t}\right)^{n} \sum_{k=0}^{n-1} \frac{(-n+1)_{k}(-1)^{k}}{(k+1) ! k ! 2^{k}} H e_{2 n+2 k+1}\left(\frac{x}{(2 t)^{1 / 2}}\right)
$$

where $H e_{n}(x)$ is a Hermite polynomial given by 


$$
H e_{n}(x)=n ! \sum_{m=0}^{|n / 2|} \frac{(-1)^{m} x^{n-2 m}}{2^{m} m !(n-2 m) !} .
$$

Some numerical experiments indicate that the first term works well for large $t$ but applies sooner for large $x$.

A similar analysis for $u_{2}(x, t)$ gives

$$
u_{2}(x, t)=\operatorname{erfc}\left(\frac{x}{2 t^{1 / 2}}\right)+e^{-x^{2 /(4 t)}}\left(\frac{2}{\pi}\right)^{1 / 2} \sum_{n=1}^{\infty}\left(\frac{\epsilon}{2 t}\right)^{n} \sum_{k=0}^{n} \frac{(-n)_{k}}{(k !)^{2}} \frac{(-1)^{k}}{2^{k}} H e_{2 n+2 k-1}\left(\frac{x}{(2 t)^{1 / 2}}\right) .
$$

Some comments on a numerical solution. Although for $\epsilon=0$ the solution of the boundary value problem (2) is closely approximated by the difference scheme

$$
\begin{gathered}
\frac{u_{i}^{n+1}-u_{i}^{n}}{\Delta t}=\frac{\Delta^{2} u_{i}^{n+1}}{(\Delta x)^{2}} \quad \begin{array}{l}
j=1,2, \cdots, N \\
n=1,2, \cdots
\end{array} \\
u_{i}^{0} \equiv 0, \quad j=1,2, \cdots N, \quad u_{0}^{n} \equiv 1, \quad u_{N+1}^{n}=0, \quad n=1,2, \cdots,
\end{gathered}
$$

the foregoing analysis shows that if the analogous difference scheme

$$
\begin{gathered}
\frac{u_{i}^{n+1}-u_{i}^{n}}{\Delta t}=\frac{\Delta^{2} u_{i}^{n+1}}{(\Delta x)^{2}}+\epsilon \frac{\Delta^{2} u_{i}^{n+1}-\Delta^{2} u_{i}^{n}}{(\Delta x)^{2} \Delta t} \quad \begin{aligned}
j & =1,2, \cdots, N \\
n & =1,2, \cdots
\end{aligned} \\
u_{i}^{0} \equiv 0, \quad j=1,2, \cdots N, \quad u_{0}^{n} \equiv 1, \quad u_{N+1}^{n}=0, \quad n=1,2, \cdots,
\end{gathered}
$$

for (2) with $\epsilon>0$ converges, it can be expected to approach $u_{2}(x, t) \cdot u_{2}(x, t)$ would also be produced by starting with

$$
u_{2}(x, 0)=e^{-x / \epsilon^{2 / 2}}, \quad u_{2}(0, t)=1 .
$$

Thus, if one wishes to produce $u_{1}(x, t)$, consideration must be given to other conditions. One expects from the analysis that either of the pairs

$$
\left\{\begin{array} { l } 
{ u _ { 1 } ( x , 0 ) = e ^ { - \alpha x } } \\
{ u _ { 1 } ( 0 , t ) = 1 }
\end{array} \quad \left\{\begin{array}{l}
u_{1}(x, 0)=0 \\
u_{1}(0, t)=1-e^{-t / \epsilon}
\end{array}\right.\right.
$$

will produce $u_{1}(x, t)$ if $\alpha$ is chosen commensurate with $\Delta x$. If $\alpha$ is too large then $u_{2}$ is produced. In practice $\alpha=1 / \Delta x$ works very well. ( $\alpha=1 / \Delta x=$ slope of the line connecting $u(0, \Delta t) \equiv 1$ with $u(\Delta x, 0) \equiv 0$.) These observations were confirmed by some numerical experiments with $\Delta x$ and $\Delta t$ as low as .001, with $\epsilon=.01, .1$ and .5 .

The proposed (implicit) difference scheme yields the usual tridiagonal system of equations for $u_{i}^{n+1}, j=1,2, \cdots, N$ which is easily solved by recursion [10, p. 198]. This numerical scheme can be expected to be unconditionally stable since for

$$
u(n, j)=\sum_{-\infty}^{+\infty} v(r, n) e^{i j r \Delta x} \quad i=(-1)^{1 / 2}
$$

the amplification factor in a von Neumann type analysis is bounded by one,

$$
0<\frac{v(r, n+1)}{v(r, n)}=\frac{(\Delta x)^{2}+4 \epsilon \sin ^{2}(r \Delta x / 2)}{(\Delta x)^{2}+4(\epsilon+\Delta t) \sin ^{2}(r \Delta x / 2)}<1 .
$$

A Taylor expansion shows the difference scheme to be consistent with the differential equation as $\Delta t$ and $\Delta x \rightarrow 0$ with an error $O(\Delta t)+O\left[(\Delta x)^{2}\right]$. On finite intervals, stability 
and consistency imply convergence, and under suitable hypotheses it seems reasonable that a convergence proof could be constructed for the infinite interval.

The difference scheme which is explicit for $\epsilon=0$

$$
\frac{u_{i}^{n+1}-u_{i}^{n}}{\Delta t}=\frac{\Delta^{2} u_{j}^{n}}{(\Delta x)^{2}}+\epsilon \frac{\Delta^{2} u_{i}^{n+1}-\Delta^{2} u_{i}^{n}}{(\Delta x)^{2} \Delta t}
$$

gives, in a similar analysis, the stability condition $(\Delta x)^{2} / 2 \geq(\Delta t-2 \epsilon)$. Thus, if $\Delta t \leq 2 \epsilon$, this is always satisfied. For $\epsilon=0$, the standard stability condition is obtained. This scheme is also consistent with the differential equation, but there is no numerical advantage over the first scheme since this is still implicit for $\epsilon>0$.

A solution for continuous boundary and initial data. For convenience we superimpose two solutions $u$ and $v$ to form $w=u+v$ where formally we solve

$$
\begin{array}{ll}
u_{t}=u_{x x}+\epsilon u_{x x t}, & v_{t}=v_{x x}+\epsilon v_{x x t}, \\
u(0, t)=\varphi(t), \quad \varphi(0)=0, & v(0, t)=f(0), \\
u(x, 0)=0, & v(x, 0)=f(x),
\end{array}
$$

and supply sufficient conditions for the existence of a continuous solution with appropriate continuous derivatives in $x \geq 0, t \geq 0$. Then $w$ satisfies

$$
\begin{aligned}
w_{t} & =w_{x x}+\epsilon w_{x x t}, \\
w(0, t) & =f(0)+\varphi(t) \quad t \geq 0, \quad \varphi(0)=0, \\
w(x, 0) & =f(x) \quad x \geq 0 .
\end{aligned}
$$

The Laplace transform for $u$ yields $\bar{u}(x, s)=\bar{\varphi}(s) e^{-a x}$ where $\bar{\varphi}(s)$ is the transform of $\varphi(t)$ and

$$
q=\left(\frac{s}{1+\epsilon S}\right)^{1 / 2}
$$

Since the exponential is not a transform, we cannot use the convolution theorem directly. Instead, we write $\bar{u}(x, s)=s \bar{\varphi}(s) e^{-a x} / s$ and then convolute

$$
u(x, t)=\int_{0}^{t} \varphi^{\prime}(\tau)\left\{1-\frac{2}{\pi} \int_{0}^{\infty} \frac{e^{-\beta^{2}(t-\tau) /\left(1+\epsilon \beta^{2}\right)} \sin \beta x d \beta}{\beta\left(1+\epsilon \beta^{2}\right)}\right\} d \tau
$$

by inverting around a contour in the upper half $s$ plane indented around the cut from $-1 / \epsilon$ to 0 . An exchange of integrals, followed by integration by parts with (5) for $t=0$, gives the candidate

$$
u(x, t)=\varphi(t) e^{-x / \epsilon^{1 / 2}}+\frac{2}{\pi} \int_{0}^{t} \varphi(\tau) \int_{0}^{\infty} \frac{e^{-\beta^{2}(t-\tau) /\left(1+\epsilon \beta^{2}\right)} \beta \sin \beta x}{\left(1+\epsilon \beta^{2}\right)^{2}} d \beta d \tau .
$$

For $v$, a formal Laplace transform solution yields

$$
v(x, t)=f(0) u_{1}(x, t)+e^{-t / \epsilon} f(x)+\frac{2}{\pi} \int_{0}^{\infty}\left[e^{-\beta^{2} t /\left(1+\epsilon \beta^{2}\right)}-e^{-t / \epsilon}\right] \bar{f}(\beta) \sin \beta x d \beta
$$

while a formal Fourier sine transform solution yields

$$
v(x, t)=f(0) u_{1}(x, t)+\frac{2}{\pi} \int_{0}^{\infty} e^{-\beta^{2} t /\left(1+\epsilon \beta^{2}\right)} \bar{f}(\beta) \sin \beta x d \beta
$$


where $\bar{f}$ is the sine transform

$$
\bar{f}(\beta)=\int_{0}^{\infty} f(\xi) \sin \xi \beta d \xi
$$

and $u_{1}(x, t)$ is taken in the form (4). These are equivalent if one assumes conditions which will ensure the inversion of $\bar{f}$ to $f$. Actually (12) is preferable since the value and limiting form for $v(x, t)$ at $x=0$ are unambiguous, the integral being uniformly convergent in the neighborhood of $x=t=0$ with only mild restrictions on $\bar{f}$. The inequality

$$
\left|e^{-\beta^{2} t /\left(1+\epsilon \beta^{2}\right)}-e^{-t / \epsilon}\right| \leq \frac{1}{1+\epsilon \beta^{2}},
$$

obtained by bounding the maximum in $t$ for fixed $v$, helps in bounding the integral independent of $x$ and $t$ for the Weierstrass $M$ test.

It is easily seen by integrating the sine transform by parts twice that two differentiations with respect to $x$ can be performed,

$v(x, t)=f(0)\left(1-e^{-t / \epsilon}\right)+f(x) e^{-t / \epsilon}-\frac{2}{\pi} \int_{0}^{\infty} \frac{\left[e^{-\beta^{2} t /\left(1+\epsilon \beta^{2}\right)}-e^{-t / \epsilon}\right]}{\beta^{2}} \bar{f}^{\prime \prime}(\beta) \sin \beta x d \beta$,

and differentiation with respect to $t$ does not hinder the convergence of the integral. However, in order to satisfy the differential equation, $f^{\prime \prime}(x) e^{-t / \epsilon}$ must be cancelled by the inversion of $e^{-t / \epsilon} \bar{f}^{\prime \prime}$. Conditions to guarantee the inversion of the Fourier integrals, in addition to

$$
\lim _{x \rightarrow 0} f(x)=\lim _{x \rightarrow 0} f^{\prime}(x)=0, \quad \varphi, \varphi^{\prime}, f^{\prime \prime} \text { continuous, }
$$

are key hypotheses for this analysis [7, pp. 12, 16].

Notice that enhancement of the convergence by adding and subtracting $f(x) e^{-t / \epsilon}$ can be done for $u_{1}(x, t)$ in (4), making a numerical quadrature more feasible:

$$
\int_{0}^{\infty}\left(e^{-\beta^{2} t /\left(1+\epsilon \beta^{2}\right)}-e^{-t / \epsilon}\right) \frac{\sin \beta}{\beta} d \beta=\sum_{n=0}^{\infty}(-1)^{n} \int_{n \pi}^{(n+1) \pi}\left[e^{-\beta^{2} t /\left(1+\epsilon \beta^{2}\right)}-e^{-t / \epsilon}\right] \frac{|\sin \beta|}{\beta} d \beta
$$

with an error bound after $N$ terms (integral truncated at $(N+1) \pi)$ asymptotic to

$$
\frac{x^{2}}{2 \epsilon \pi^{2}} \cdot \frac{2 N+3}{(N+1)^{2}(N+2)^{2}} \cdot
$$

Then

$$
u_{1}(x, t)=1-e^{-t / \epsilon}-\frac{2}{\pi} \sum_{n=0}^{\infty}(-1)^{n} \int_{n \pi}^{(n+1) \pi}\left[e^{-\beta^{2} t /\left(1+\epsilon \beta^{2}\right)}-e^{-t / \epsilon}\right] \frac{|\sin \beta|}{\beta} d \beta .
$$

It is worthwhile to note that the alternating series bound retains $O\left(1 / N^{3}\right)$ convergence while a direct estimate for the truncation error in the form

$$
x^{2} \int_{(N+1) \pi}^{\infty} \frac{d \beta}{\beta\left(x^{2}+\epsilon \beta^{2}\right)}
$$

gives $O\left(1 / N^{2}\right)$ convergence. 


\section{REFERENCES}

[1] G. I. Barenblatt, Iu. P. Zheltov and I. N. Kochina, Basic concepts in the theory of seepage of homogeneous liquids in fissured rocks [Strata], PPM 24, 1286-1303 (1960) (Translation of Prikl. Mat. i Meh.)

[2] P. J. Chen and M. E. Gurtin, On a theory of heat conduction involving two temperatures, Z. Angew. Math. Phys. 19, 614-627 (1968)

[3] B. D. Coleman, R. J. Duggin and V. J. Mizel, Instability, uniqueness, and non-existence theorems for the equation $u_{t}=u_{x x}-u_{x t x}$ on a strip, Arch. Rational Mech. Anal. 19, 100-116 (1965)

[4] B. D. Coleman and W. Noll, An approximation theorem for functionals with applications in continuum mechanics, Arch. Rational Mech. Anal. 6, 355-370 (1960)

[5] A. Erdélyi et al., Tables of integral transforms. Vol. 1, Bateman Manuscript Project, McGraw-Hill, New York, 1954

[6] W. Gautschi, Computational aspects of three-term recurrence relations, SIAM Rev. 9, No. 1, 24-83 (1967)

[7] R. R. Goldberg, Fourier transforms, Cambridge Univ. Press, New York, 1962

[8] M. E. Gurtin and W. O. Williams, An axiomatic foundation for continuum thermodynamics, Arch. Rational Mech. Anal. 26, 83-117 (1967)

[9] M. E. Gurtin and W. O. Williams, On the Clausius-Duhem inequality, Z. Angew. Math. Phys. 17, 626-633 (1966)

[10] R. D. Richtmyer and K. W. Morton, Difference methods for initial-value problems, 2nd Ed., Interscience, New York, 1967

[11] E. C. Titchmarsh, Theory of functions, 2nd Ed., Oxford Univ. Press, London, 1939 